\title{
BMJ Open Quality Initiative to improve the cardiogenic safety of antipsychotic medication in community mental health patients
}

\author{
Katherine Margaret Parks, ${ }^{1}$ Fiona Donnelly, ${ }^{2}$ Jane Smithies ${ }^{2}$
}

To cite: Parks KM, Donnelly F, Smithies J. Initiative to improve the cardiogenic safety of antipsychotic medication in community mental health patients.BMJ Open Quality 2017;6:e000223. doi:10.1136/ bmjoq-2017-000223

- Additional material is published online only. To view please visit the journal online (http://dx.doi.org/10.1136/ bmjoq-2017-000223).

Received 27 September 2017 Revised 31 October 2017 Accepted 1 November 2017

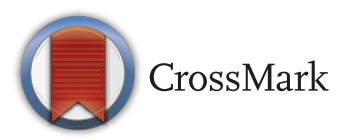

${ }^{1}$ Pharmacy, Greater Manchester Mental Health NHS Foundation Trust, Manchester, UK

${ }^{2}$ Greater Manchester Mental Health NHS Foundation Trust, Manchester, UK

Correspondence to Katherine Margaret Parks; kparks02@qub.ac.uk

\section{ABSTRACT}

Serious mental illness is reported to reduce a patient's life expectancy by 15-20 years. This disparity is thought to be related to lifestyle factors, access to healthcare, poor health monitoring and the common use of antipsychotics, which can cause serious metabolic and cardiogenic side effects. Therefore, to reduce the risk of cardiac complications, both national and local guidance recommends annual ECG monitoring for patients on antipsychotics. Unfortunately this monitoring is not completed consistently at Manchester Mental Health and Social Care Trust, especially within community mental health teams. A small team of healthcare professionals conducted a quality improvement (QI) project from June 2015 to May 2016 with the aim of addressing this deficiency in care. A multidisciplinary approach was used to implement improvement in four key areas. Awareness of the need for monitoring, patient engagement with this process, identification of patients requiring monitoring and access to ECG equipment were all addressed as separate primary drivers for change over an 8-month period using a 'Plan Do Study Act' model of QI. Outcome, process and balancing measures were gathered monthly to track progress and improvement following the application of change. Compliance with annual ECG monitoring nearly doubled throughout the course of the project from 43\% in June 2015 to a peak of 83\% in February 2016. Improvement appeared to be sustained as the percentage of patients receiving the required monitoring remained significantly higher than baseline even after no further change interventions were being implemented $(76 \%, 71 \%$, $77 \%$, March, April, May 2016). This QI project has shown that improvements can be made and has documented a recipe for how this change was achieved.

\section{PROBLEM}

The disparity between mental and physical healthcare is wide-ranging and nationally recognised. There have been several national calls to action to improve the physical health of patients with serious mental illness (SMI) and reduce the gap in terms of inequality. ${ }^{1}{ }^{2}$ National steering groups, government reports, and national and local Commissioning for Quality and Innovation targets all support this goal. However, despite this national drive, Manchester Mental Health and Social Care Trust (MMHSCT) recognised that it was failing to adequately monitor some of its patients on antipsychotics, especially patients who are managed under community mental health teams (CMHTs). MMHSCT provides care in the community to patients with SMI via six large CMHTs within the central Manchester area. Each team holds a case load of approximately 500 patients, and therefore the inconsistent cardiac monitoring of patients on antipsychotics could be impacting on a very significant number of patients within Manchester. Consequently MMHSCT began a yearlong quality improvement (QI) project in one of the CMHTs with the aim of improving the cardiogenic safety of antipsychotic medication in community mental health patients by ensuring more patients received annual ECG monitoring.

The following were the key objectives:

- To double the percentage of patients in the South Mersey Community Mental Health Team (SM CMHT) who receive yearly QTc monitoring if prescribed any antipsychotic by March 2016 from 43\% to $86 \%$.

- To halve the percentage of patients in SM CMHT on an antipsychotic who have never received an ECG by March 2016.

- To ensure that $100 \%$ of QTc prolongation detected during ECG monitoring was treated appropriately.

\section{BACKGROUND}

Most antipsychotics used within psychiatry may cause QTc prolongation by blocking cardiac potassium channels and delaying repolarisation. This prolongation of the QTC interval is a risk factor for the ventricular arrhythmia Torsades de Pointes (TdP), which can be fatal. ${ }^{34}$ The true incidence of TdP is largely unknown partially due to under-reporting. A large epidemiological study in Germany estimated that the rate of TdP may be somewhere between 2.5 and 4 cases per million per year in the general population, ${ }^{5}$ but this rate would be significantly higher in patients prescribed antipsychotics. It is estimated that there are 15 cases of sudden 
cardiac death per 10000 years of drug exposure in patients on antipsychotics, although the exact incidence of TdP is unknown because of diagnostic uncertainty. ${ }^{6}$

TdP occurs almost exclusively in patients with a prolonged QTc interval, but the relationship between QTc and TdP is complex as arrhythmia risk is also affected by other factors, such as existing heart disease and electrolyte disturbances. However there is fairly convincing evidence that a QTc interval over $500 \mathrm{~ms}$ is associated with a significantly increased risk of arrhythmia. ${ }^{7}$ The use of most antipsychotics may be associated with an increased risk of sudden cardiac death probably due to their arrhythmogenic potential. Therefore although QTc measurement via an ECG is not a clear indicator of arrhythmia risk, it remains an important estimate of arrhythmia risk and sudden cardiac death risk.

To help manage this risk, the locally agreed Shared Care Protocol (SCP) requires that all patients on an antipsychotic have annual ECG monitoring, and the National Institute for Health and Care Excellence clinical guidelines recommend that an ECG is conducted prior to prescribing an antipsychotic for all inpatients and patients with other risk factors. ${ }^{89}$ However it is widely accepted that ECG monitoring within a mental health setting is complicated by a number of factors including the following: psychiatrists may not feel competent in ECG interpretation, ECG devices may not be as readily available within mental health settings compared with general medical settings, and finally ECG monitoring may be difficult to perform in acutely unwell patients.

\section{BASELINE MEASUREMENT}

Data was initially collected in June 2015 to gain an understanding of the extent of the suspected problem. A sample of 50 patients were selected from the SM CMHT case load using a random number generator. A pro forma was developed and faxed to all of the patients' general practitioner (GP) surgeries to clarify whether they were prescribed an antipsychotic, and if so when their last ECG was conducted. Trust notes were also accessed for each patient to determine whether MMHSCT was prescribing any antipsychotics directly and when the patient's last ECG was documented. Completion rate of the pro forma was initially low but improved after several prompts. Of the 39 patients with completed pro formas, 28 of them were prescribed a regular antipsychotic, but only 12 (43\%) had had an ECG within the past year. No ECG had ever been recorded in primary or secondary care for six patients $(22 \%)$ on antipsychotics despite a baseline and annual ECG being a requirement of the Shared Care Protocol. In order to achieve our project aim, we continued to collect and chart these measures on a fortnightly basis to guide QI actions, and we decided to collect the information directly from GP surgeries to help build good working relationships and improve pro forma completion rate. Management of abnormal ECG results was added as an important outcome measure, and recent cessation of antipsychotic therapy was included as a balancing measure to ensure that any change in this area of the system was considered when analysing any change. Of the patients who had had an ECG during the baseline data collection, most of these had been done at the GP surgery $(67 \%)$, and only $17 \%$ had been done by outpatient psychiatry, and this information was used to direct our QI actions during the project.

\section{DESIGN}

It was clear from the baseline results that the scope and extent of the problem required a multitargeted approach; therefore, a multidisciplinary QI team was formed including a range of healthcare professionals, front-facing and corporate staff. This included a consultant psychiatrist, team manager, assistant practitioner, community psychiatric nurse, pharmacy technician and pharmacist. Four key primary drivers for change were quickly identified: staff awareness, identification, access and patient engagement. With the agreement of our key stakeholders, these primary drivers were incorporated into a driver diagram with all of the secondary drivers and change concepts focusing on these four key issues.

\section{Staff awareness}

Awareness of staff both in MMHSCT and primary care was targeted throughout the project by poster and flashcard distribution, meeting attendance, training sessions, presentations and incident reporting. A guidance document detailing the appropriate management of QTc prolongation was developed to help support primary care staff handle any abnormal results and increase confidence. Data was collected directly from GP surgeries by the QI team, giving us the opportunity to raise awareness of the issue and the Shared Care Protocol personally with GP staff while collecting data.

\section{Identification}

A centralised database was set up within the SM CMHT to help identify which patients were on an antipsychotic and when they were due an ECG. A protocol was drafted to maintain this database monthly, prompting any patients who were due an ECG that month, adding any patients who had received an ECG, removing any discharged patients and adding any new admissions.

\section{Access}

With assistance and guidance from cardiology in a neighbouring acute Trust, a portable ECG device was purchased, risk-assessed and implemented within the SM CHMT to help address issues with access to ECG equipment.

\section{Patient engagement}

Targeted processes were implemented to optimise patient engagement following extensive Plan Do Study Act (PDSA) tests. 


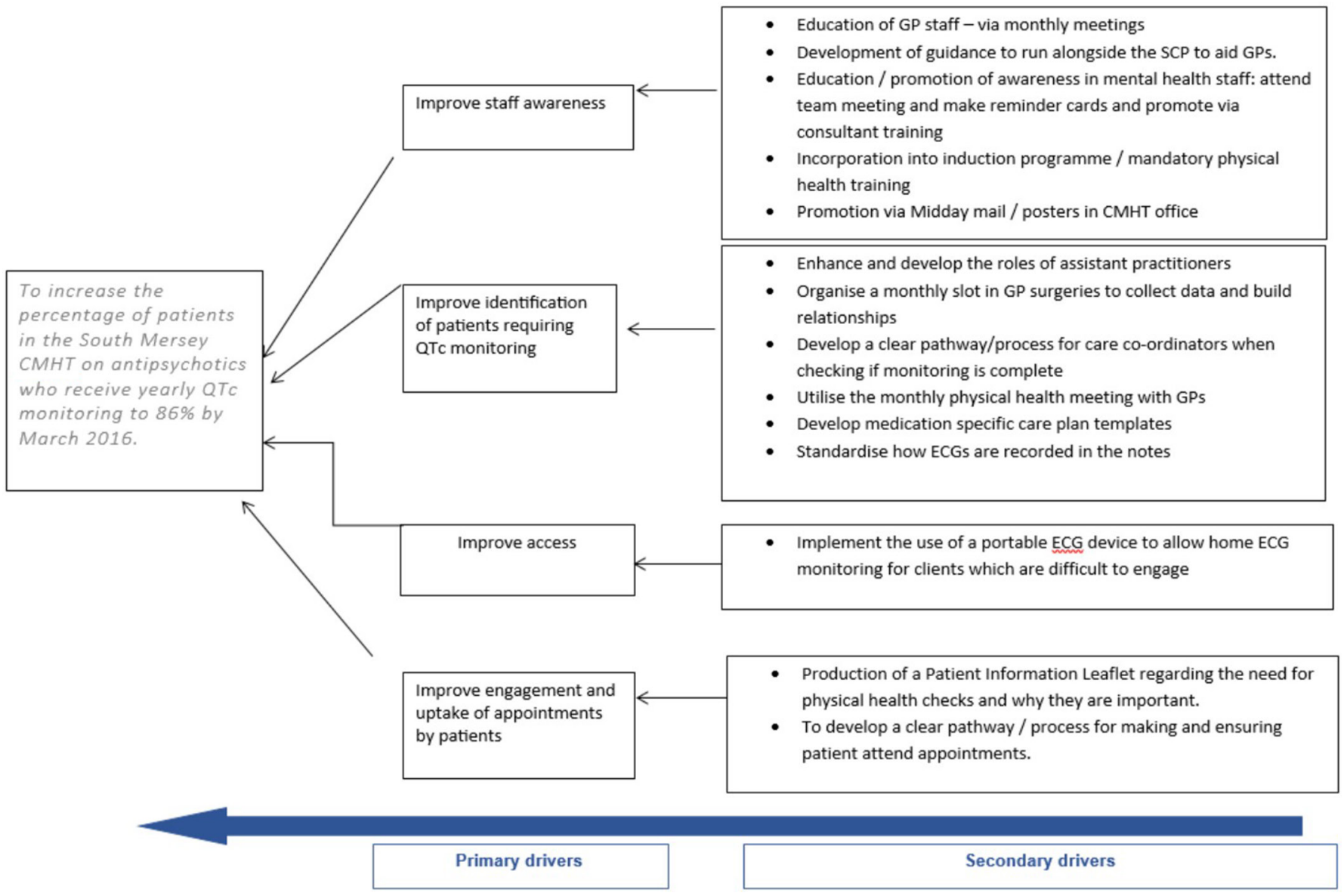

Figure 1 Driver diagram outlining the changes implemented during the initiative. $\mathrm{CC}$, care co-ordinator; $\mathrm{CMHT}$, community mental health team; GP, general practitioner; MH, mental health; PIL, patient information leaflet.

The QI team met every fortnight during the initial stages of the project to review progress, track change and help the initiative gain momentum.

\section{STRATEGY}

The Specific Measurable Achievable Realistic Time-limited (SMART) aim of our QI project was to increase the percentage of patients in the SM CMHT on antipsychotics who received yearly QTc monitoring to $86 \%$ by March 2016 . Our strategy for improvement was collated into a driver diagram that included primary drivers, secondary drivers and concepts for change. This driver diagram (figure 1) formed the agenda of our team meetings and was continually reviewed throughout the project based on the results of each PDSA cycle and any new ideas.

Numerous PDSA cycles were conducted for each of the primary drivers, with different PDSA cycles often happening simultaneously. This approach allowed many different changes ideas to be trialled and tested numerous times before implementation; however, it makes it more difficult to link improvement to specific changes that were implemented.

1. One of the first PDSA cycles that we undertook was focused on staff awareness as this was initially thought to be a straightforward and quick driver to affect change (see online supplementary 1). A clinical pharmacist spoke at the SM CHMT meeting and distributed information to all care coordinators via email with the aim of improving the awareness of the need for annual ECG monitoring. Awareness in the team was then gauged via informal questioning and completion of a brief questionnaire. Awareness did improve following this intervention; however, it also highlighted a training need within the team, as some staff, especially non-nurses, were unsure which medications were classed as antipsychotics. The PDSA was repeated, but in addition to general awareness information was given on which drugs are antipsychotics and what monitoring is required via laminated diary cards, posters and inclusion in the physical health e-learning. The PDSA structure was used to hone the diary cards and posters prior to implementation to the wider team.

2. The next logical PDSA cycle after improving MMHSCT staff awareness was to increase the awareness of our primary care colleagues (see online supplementary 1). Meetings were set up between a clinical pharmacist and senior primary care representatives to discuss the SCP and ECG monitoring, with the aim of improving awareness. This cycle was unsuccessful and did 
not improve awareness as awareness was already high among this cohort of healthcare professionals. A variation of this PDSA cycle was repeated with other primary care professionals during data collection visits to check whether this method could be used to improve awareness within different cohorts of professionals and was successful. After repeating several times, this was implemented across the SM patch. The lack of guidance regarding the management of possible antipsychotic-induced QTc prolongation and lack of patient engagement were both highlighted as a barrier to compliance during this PDSA and were investigated during subsequent cycles. A guidance document that detailed action to be taken to address QTc prolongation in patients on psychotropics was drafted in combination with a cardiology team and tested using the PDSA cycle format until a final version was agreed and implemented throughout the Trust to support primary care staff in managing this situation.

3. Identification of patients who were due an ECG by care coordinators was highlighted as an issue at an early stage of the initiative. A central SM CHMT database that detailed when patients were due an ECG was tested with the hope of aiding identification of patients who needed monitored. The initial PDSA cycle did not result in significant changes in monitoring compliance as predicted. Several PDSA cycles were repeated on the database, including new processes for admitted patients, discharged patients and medication changes. Finally following rigorous testing, a database was agreed and a local procedure was implemented regarding the management of that database.

4. Patient engagement with physical health monitoring can be challenging. Four different change concepts were tested to try and improve patient engagement. (1) A letter was sent to the patient asking them to make an appointment for an ECG. (2) The patient was phoned and asked to make an appointment for an ECG at their GP surgery. (3) An ECG appointment was made for the patient and they were phoned to inform them of the appointment. (4) A duplicate of (3) but the patient was also called the day before the appointment to remind them. After testing it was found that options (3) and (4) were equally effective; therefore, this process for ensuring patients attended ECG appointments was implemented throughout SM CMHT and patients decided whether they wanted a reminder phone call. Building on the success of these PDSA cycles, it was decided to test whether informing patients why ECGs are important and necessary could even further increase engagement. A member of the pharmacy team explained the rationale for ECG testing and why it is important when informing patients of their appointment. This did improve patient uptake; however, sufficient resources were not available for pharmacy staff to offer this service consistently; therefore, a patient information leaflet was developed as an alternative way to convey this information to patients, tested via PDSA structure and implemented.

5. Previous PDSA cycles had shown that despite the staff's best efforts in improving patient engagement and appointment uptake, there were a small number of patients who were unable or unwilling to attend the GP surgery or outpatient clinic for an ECG. A portable ECG device-the AliveCor heart monitor-was riskassessed, purchased and trialled to determine whether access to an ECG device at home for these patients could improve monitoring compliance. The initial PDSA cycles were successful but highlighted issues around the interpretation of the ECG traces produced. Guidance to aid interpretation of these one-lead ECG traces was developed in combination with cardiology, and following approval was implemented alongside guidance for use of the device in the SM CHMT only for patients who otherwise would be unable to receive an ECG.

\section{RESULTS}

Our main outcome measure was to increase the percentage of clients on an antipsychotic in SM CMHT who had received an ECG in the past year, and as a result of our initiative this figure nearly doubled from baseline to March 2016 (see figure 2). Our secondary outcome measure was to halve the percentage of patients on an antipsychotic who had never had an ECG.

Three main probability-based rules-shift, trend and runs-were used to analyse the run chart data for both of our outcome measures and detect statistical significance based on an alpha level of $0.05^{10}$ : shift: six or more consecutive data points plotted above or below the median on a run chart show a statistically significant difference; trend: five or more points all ascending or descending show statistical significance; and runs: statistical significance can be detected on run charts by too few or too many runs crossing the median using critical values based on the total number of data points that do not fall on the median. ${ }^{10}$ On analysis of the run charts, both outcome measures show statistical significance based on the shift probability-based rule. Eight consecutive data points plotted above the population median on the run chart show that the initiative made a significant and sustained improvement on annual ECG compliance. The percentage of clients on an antipsychotic who had never had an ECG nearly halved during the intervention period. Eight consecutive data points below the population median show this change to be statistically significant and sustained (see figure 3). This parameter took several months to improve as often patients who have never had an ECG are those who take the longest to engage, and therefore if this initiative was run over a longer time frame there may have been an even bigger long-term improvement noted.

Approximately 3\% of all ECGs completed showed QTc prolongation defined as $>470 \mathrm{~ms}$ for women and $>440 \mathrm{~ms}$ for men. ${ }^{3}$ The rate of QTc prolongation described in the 


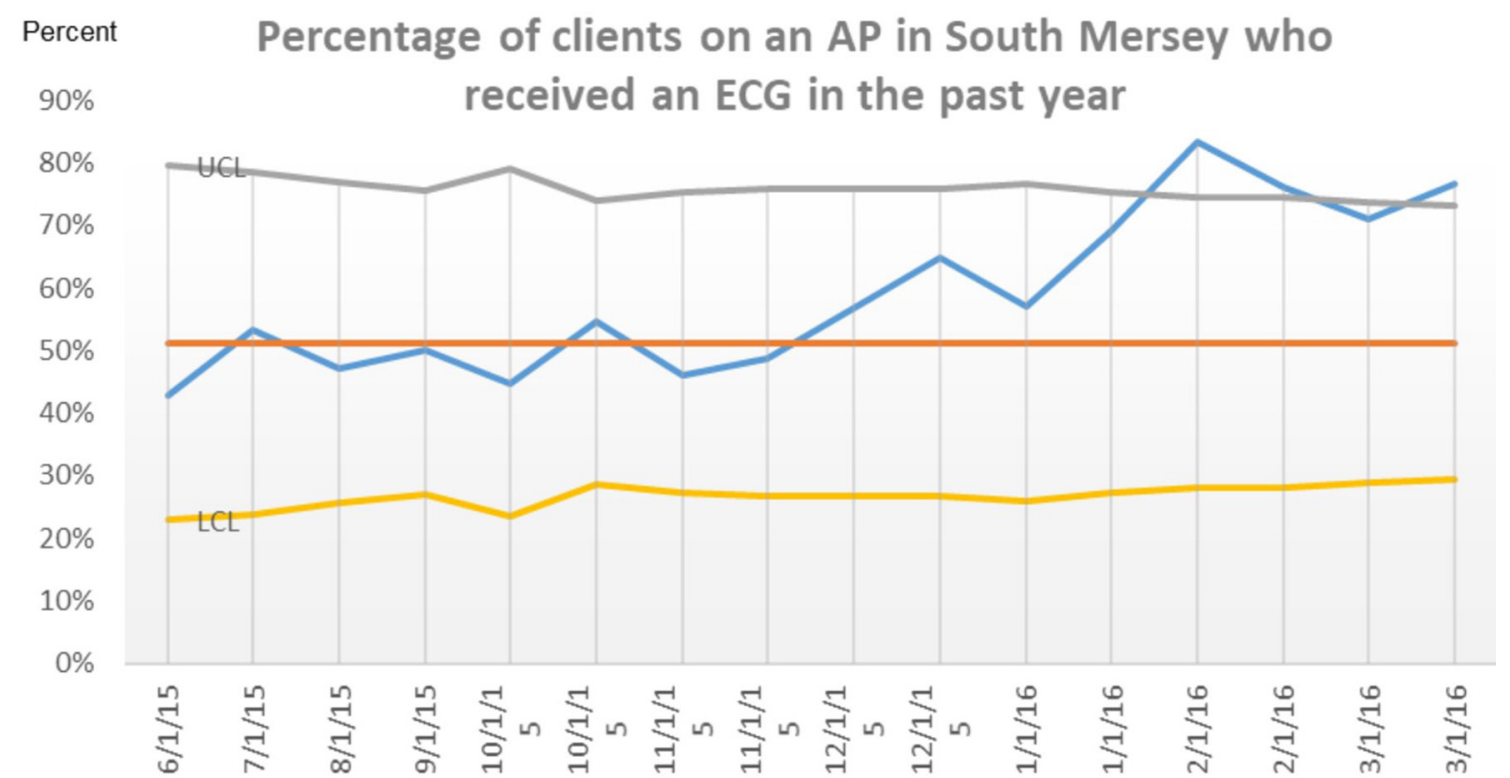

Figure 2 A graph showing the change in percentage of patients on an antipsychotic (AP) who received an annual ECG.

literature varies widely due to the numerous patient-related factors that can affect the risk and values quoted range from between $0.06 \%$ and $8 \% .^{11} 12$ Therefore the findings in this QI project are in line with other current literature. Any ECGs that highlighted QTc prolongation were dealt with appropriately as per the Trust-approved guidance, which involved reviewing and removing any modifiable risk factors, repeating the measurement and seeking specialist cardiology advice for a risk:benefit analysis if appropriate.

The rate of discontinuation of antipsychotics was analysed as a balancing measure throughout the project to reflect what may be happening elsewhere in the system as a result of the changes. However, it remained stable at $1 \%-2 \%$ throughout the 10 months so will not have skewed the results in any way.

\section{LESSONS AND LIMITATIONS}

Due to time constraints and the time it takes from deciding a patient needs an ECG to one being completed (several weeks in some cases due to delays in available appointments and poor patient engagement), numerous PDSA cycles and change concepts were trialled simultaneously. This makes it more difficult to compare different initiatives and their relative effect on the project outcome. Knowing which interventions had more of an effect than other interventions would help future projects prioritise their time. If this project had been run over 2 years rather than 10 months, there would have been sufficient time to implement just one initiative at a time and measure any improvement as a result of this change for several months to account for the lag period.

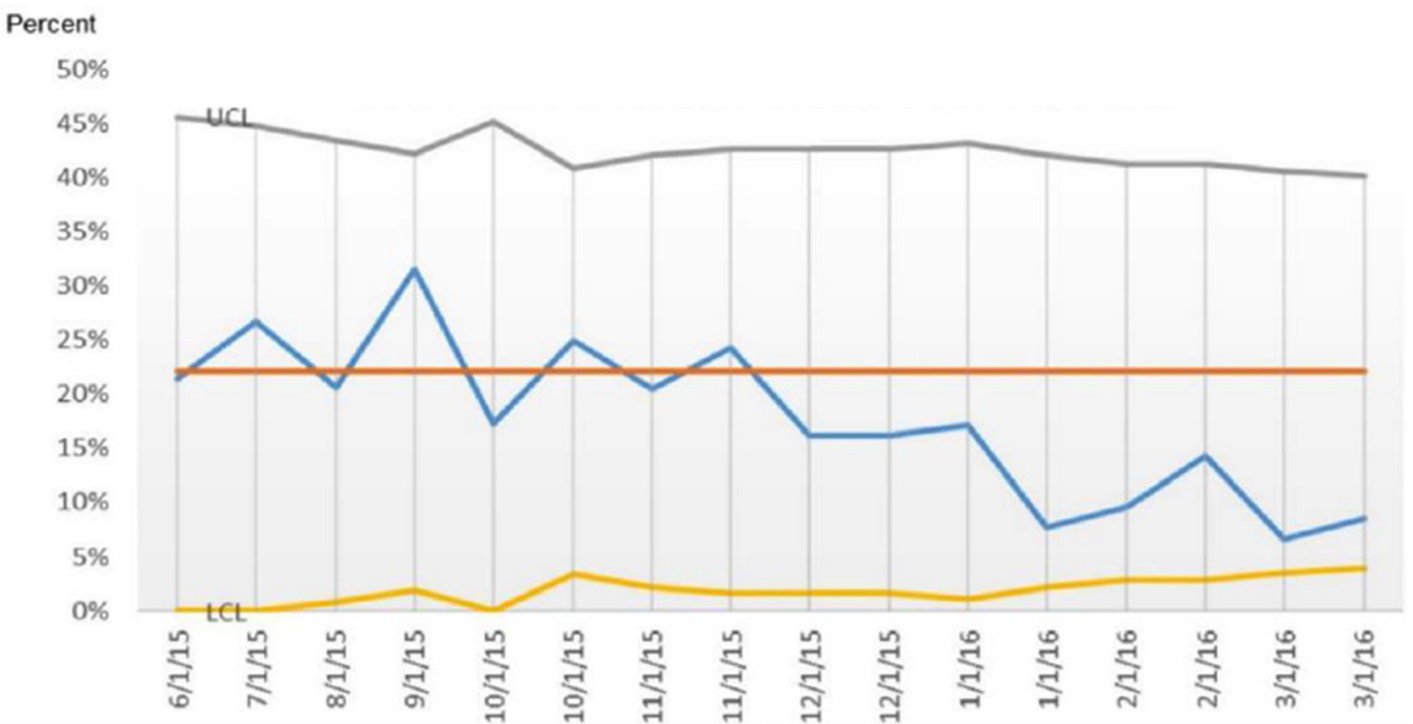

Figure 3 A graph showing the change in the percentage of patients on antipsychotics (AP) who had never had an ECG. 
There was a significant lag between changes being implemented within the system and the primary outcome improving. This is partly explained by the length of the ECG process; however, a general resistance to change was also a likely contributing factor. An assistant practitioner from the SM CMHT was a core member of the project team; however, no other frontline SM CMHT staff were part of the core team, and this may have made it more difficult to affect change within the CMHT. Appropriate team selection is vital to the success of a QI project, and more front-line staff and more directorate-level staff inclusion in the team may have enabled the initiative to progress more quickly as directorate buy-in would have enabled changes to progress quicker when approval or a change in procedure was required.

From the time-tracked data, it is clear that during this initiative, there was no single intervention that had a really significant impact on ECG compliance in isolation, rather compliance gradually increased with time as more and more interventions were trialled and implemented. This shows that to effect significant change in this area, a multitargeted and sustained approach is required and that individual initiatives in isolation are unlikely to be successful.

All of the successful interventions trialled during this project were implemented into routine practice to ensure the improvement achieved would be sustained. Including a key member of the front-line team in our project team significantly helped with sustainability of this project as he was able to assist with implementation and continued to use and update the patient identification database regularly as part of routine clinical practice even after the project had finished. The QI project team tried to engage various members of the SM CMHT in conducting PDSA cycles throughout the project, including the PDSAs that investigated patient engagement. Several different care coordinators participated in this test of change and began to implement the most successful strategies immediately when trying to get patients to attend ECG appointments. Involvement of these key front-line staff from the beginning of the project helped with the momentum of the project but also helped ensure sustainability of the improvement.

\section{CONCLUSION}

It is well known that antipsychotics may cause QTc prolongation and affect the cardiogenic safety of the patients who take them. Ensuring this risk is minimised and managed is part of our duty of care as healthcare professionals and NHS Trusts. This QI project achieved its aim of improving ECG monitoring in patients on antipsychotics; however, the change concepts that were applied and the processes that were conducted could be mirrored to improve compliance with nearly any other physical health intervention in this cohort of patients. Although there were some limitations to this project, the measures used were entirely appropriate and the quantity and quality of data presented are sufficient to allow conclusions to be drawn from this work. The detailed driver diagram that was used to steer this initiative should allow scale-up and spread to other CMHTs within MMHSCT and to other services within the Trust and hopefully even other MH Trusts. This project will be used as part of a business case to promote similar change within another area of the Trust and will be presented at a national mental health pharmacy conference with the aim of sharing good practice and ultimately protecting the cardiogenic health of as many patients as possible.

Acknowledgements Thanks to all the members of the QI team—Michelle Cleary, Urgent Care Pharmacy Technician; Jane Smithies, Senior Pharmacy Technician, MMHSCT; FD, Consultant Psychiatrist, MMHSCT; Stuart Freedson, Assistant Practitioner, MMHSCT; and Petra Brown, Chief Pharmacist, MMHSCT—and with special thanks to the Improvement Science for Academics (ISA4c) team at Haelo and the cardiology department at University Hospital of South Manchester for their input and expert advice. Thanks to Lesley Smith, Chief Pharmacist PCFT, for her encouragement and support.

Contributors KP was in charge of the planning, conduct and reporting of the work described in the article and is responsible for the overall content as the guarantor. FD and JS as coauthors made substantial contributions to the project, including planning, conduct, drafting the article and approval of the published version.

Competing interests None declared.

Provenance and peer review Not commissioned; externally peer reviewed.

Open Access This is an Open Access article distributed in accordance with the Creative Commons Attribution Non Commercial (CC BY-NC 4.0) license, which permits others to distribute, remix, adapt, build upon this work non-commercially, and license their derivative works on different terms, provided the original work is properly cited and the use is non-commercial. See: http://creativecommons.org/ licenses/by-nc/4.0/

(C) Published by the BMJ Publishing Group Limited. For permission to use (where not already granted under a licence) please go to http://www.bmj.com/company/ products-services/rights-and-licensing/

\section{REFERENCES}

1. Royal College of Psychiatrists. Improving the physical health of adults with severe mental illness: essential actions. $2016 \mathrm{http} / / / \mathrm{www}$. rcpsych.ac.uk (accessed Aug 2017).

2. National Association of State Mental Health Program Directors (NASMHPD) Medical Directors Council. Morbidity and mortality in people with serious mental illness. 2006 https://www.nasmhpd.org/ (accessed Aug 2017).

3. Taylor D, Paton C, Kapur S. The maudsley prescribing guidelines. 12th edn. Oxford: Wiley-Blackwell, 2015.

4. Bazire S. Psychotropic Drug Directory 2016. 27th edn: LloydReinhold Publications Ltd, 2016.

5. Sarganas G, Garbe E, Klimpel A, et al. Epidemiology of symptomatic drug-induced long QT syndrome and torsade de pointes in Germany. Europace 2014;16:101-8.

6. Nielsen J, Graff C, Kanters JK, et al. Assessing QT interval prolongation and its associated risks with antipsychotics. CNS Drugs 2011;25:473-90.

7. CredibleMeds. Overview of long QT syndrome and torsades de pointes. 2015 http://www.crediblemeds.org (accessed Aug 2017).

8. Brown P. Shared care protocol for atypical antipsychotics: Mental Health and Social Care Trust, 2013.

9. National Institute for Health and Clinical Excellence. Psychosis and schizophrenia inadults: treatment and management, clinical guideline 178. 2014 http://www.nice.org.uk.

10. Perla RJ, Provost LP, Murray SK. The run chart: a simple analytical tool for learning from variation in healthcare processes. BMJ Qual Saf 2011;20:46-51.

11. Reilly JG, Ayis SA, Ferrier IN, et al. QTc-interval abnormalities and psychotropic drug therapy in psychiatric patients. Lancet 2000;355:1048-52.

12. Beach SR, Celano CM, Noseworthy PA, et al. QTc prolongation, torsades de pointes, and psychotropic medications. Psychosomatics 2013;54:1-13. 